\title{
Electroweak phase transition in the real triplet extension of the SM: Dimensional reduction
}

\author{
Lauri Niemi, ${ }^{1, *}$ Hiren H. Patel, ${ }^{2, \dagger}$ Michael J. Ramsey-Musolf,, ${ }^{2, \nLeftarrow}$ Tuomas V. I. Tenkanen, ${ }^{1, \S}$ and David J. Weir ${ }^{1, \|}$ \\ ${ }^{1}$ Department of Physics and Helsinki Institute of Physics, University of Helsinki, \\ PL 64, Helsinki FI-00014, Finland \\ ${ }^{2}$ Amherst Center for Fundamental Interactions, Department of Physics, University of Massachusetts, \\ Amherst, Massachusetts 01003, USA
}

(Received 7 May 2019; published 5 August 2019)

\begin{abstract}
In a series of two papers, we make a comparative analysis of the performance of conventional perturbation theory to analyze electroweak phase transition in the real triplet extension of the Standard Model ( $\Sigma \mathrm{SM})$. In Part I (this paper), we derive and present the high- $T$ dimensionally reduced effective theory that is suitable for numerical simulation on the lattice. In Part II, we will present results of the numerical simulation and benchmark the performance of conventional perturbation theory. Under the assumption that $\Sigma$ is heavy, the resulting effective theory takes the same form as that derived from the minimal Standard Model. By recasting the existing nonperturbative results, we map out the phase diagram of the model in the plane of triplet mass $M_{\Sigma}$ and Higgs portal coupling $a_{2}$. Contrary to conventional perturbation theory, we find regions of parameter space in which the phase transition may be first order, second order, or crossover. We comment on prospects for prospective future colliders to probe the region where the electroweak phase transition is first order by a precise measurement of the $h \rightarrow \gamma \gamma$ partial width.
\end{abstract}

DOI: $10.1103 /$ PhysRevD.100.035002

\section{INTRODUCTION}

Explaining the origin of the observed baryon asymmetry of the Universe, characterized by the baryon-to-entropy density ratio [1],

$$
Y_{B} \equiv \rho_{B} / s=(8.61 \pm 0.09) \times 10^{-11},
$$

remains an outstanding problem at the interface of highenergy and nuclear physics with cosmology. General considerations identified by Sakharov [2] impose three criteria on early Universe particle physics in order to explain the asymmetry: nonconservation of baryon number, violation of $C$ and $C P$ invariance, and the presence of nonequilibrium conditions. ${ }^{1}$ While the Standard Model (SM) of particle physics supplies the baryon nonconserving interactions in the form of sphaleron processes, it provides neither the requisite nonequilibrium conditions nor sufficiently effective

\footnotetext{
*lauri.b.niemi@helsinki.fi

†hhpatel@umass.edu

${ }^{\ddagger}$ mjrm@physics.umass.edu

\$tuomas.tenkanen@helsinki.fi

"david.weir@helsinki.fi

${ }^{1}$ The latter requirement assumes $C P T$ invariance.
}

Published by the American Physical Society under the terms of the Creative Commons Attribution 4.0 International license. Further distribution of this work must maintain attribution to the author(s) and the published article's title, journal citation, and DOI. Funded by SCOAP ${ }^{3}$.
$C P$ violation. Thus, physics beyond the Standard Model (BSM) is essential.

Several mechanisms that satisfy the required criteria have been advanced. Among the most compelling and theoretically well motivated is electroweak baryogenesis, wherein the baryon asymmetry is generated during the era of electroweak symmetry breaking (EWSB) (for a recent review, see Ref. [3]). Successful baryogenesis requires that symmetry breaking occurred due a strongly first-order electroweak phase transition (EWPT). Numerical lattice simulations [4-9] indicate that EWSB in the SM occurred through a crossover transition for a Higgs mass at its observed value of $125 \mathrm{GeV}$ [10,11], suggesting that the Universe never departed from thermal equilibrium during this epoch.

BSM scenarios may alleviate this SM shortcoming through the addition of an extended scalar sector. The latter may catalyze a strong first-order electroweak phase transition (SFOEWPT) through new loop corrections to the zero-temperature $(T)$ Coleman-Weinberg potential, thermal loop corrections to the finite- $T$ effective potential, a modification of the tree-level vacuum structure of the theory, or a combination involving more than one of these effects. The result may be not only a SFOEWPT to the present "Higgs phase" but also a richer pattern of symmetry breaking that precedes the Higgs phase than one obtains in the SM.

These possibilities have been explored in both UVcomplete theories, such as the minimal supersymmetric 
Standard Model (MSSM), and simplified models that consider only the extended scalar sector. While simplified models are not realistic descriptions of nature, their use allows one to identify general features of phase transition dynamics that may occur in various UV-complete theories and to delineate the corresponding phenomenological consequences. Perhaps the most widely considered such simplified model involves the addition of a real scalar that carries no SM gauge charge. The phase transition dynamics of the singlet-extended Standard Model (xSM) and corresponding implications for high-energy collider experiments have been studied in Refs. [12-19]. A variant with a complex singlet (cxSM) has been analyzed in Refs. [20,21]. The viability of a SFOEWPT arising from scalars charged under $\mathrm{SU}(3)_{C}$ (including, e.g., light stops in the MSSM) is severely constrained by the nonobservation of these particles at the LHC as well as by the measured Higgs boson signal strengths $[22,23]$.

The constraints on colorless electroweak multiplets are considerably weaker. Here, we consider the colorless electroweak multiplet containing the fewest degrees of freedom (d.o.f.), the real triplet $\Sigma$ that has vanishing hypercharge. The collider phenomenology and EWPT dynamics of the " $\Sigma \mathrm{SM}$ " have been considered in Refs. [24,25]. The finite- $T$ history of the $\Sigma$ SM includes the possibility of two-step EWSB, in which-prior to entering the Higgs phase-the Universe enters a phase of broken electroweak symmetry involving a nonvanishing vacuum expectation value (vev) for the neutral component of $\Sigma$ but a vanishing neutral Higgs vev. The transition to the $\Sigma$ phase can be strongly first order, a possibility that is presently less constrained phenomenologically than a single-step SFOEWPT to the Higgs phase. The possibility of baryogenesis during the first step of the two-step scenario has been explored in Ref. [26]. For a general analysis of the two-step EWSB scenario, see Ref. [27].

The foregoing studies in the xSM, cxSM, $\Sigma S M$, and even the two-Higgs-doublet model have employed perturbation theory $^{2}$ [29-32]. But general considerations imply that the perturbative expansion formally breaks down due to enhanced infrared behavior in the vicinity of a phase transition [33,34]. Indeed, the existence of a crossover transition and the presence of a critical point in the SM have only been observed in computations based on lattice simulations and not in perturbative studies. Nonetheless, perturbative computations in both the SM and MSSM indicate reasonable qualitative if not quantitative agreement with other features of nonperturbative computations, such as the dependence of thermodynamic properties on the underlying model parameters.

With an eye toward a more robust assessment of the viability of a SFOEWPT (one or two step) in the $\Sigma$ SM, we

\footnotetext{
${ }^{2}$ However, see Ref. [28] for a recent nonperturbative study of the two-Higgs doublet following a methodology similar to that in this paper.
}

present in this paper a first step toward "benchmarking" the existing perturbative analyses. We do so in two parts. First, we derive the dimensionally reduced, three-dimensional effective field theory (DR3EFT) that are most amenable to lattice simulations. Depending on the mass of $\Sigma$, we derive matching relations between the effective field theory (EFT) parameters and those of the full theory. Assuming the triplet $\Sigma$ is heavy or superheavy (defined in Sec. III below) where it is integrated out, we utilize the results of existing lattice computations for the DR3EFT in which the Higgs boson is the only dynamical scalar to analyze the nature of the single-step transition to the Higgs phase. While this case cannot address the viability of the two-step EWSB scenario since the $\Sigma$ has been integrated out, it does provide one arena in which to compare with the corresponding perturbative calculations. Assessing the dynamics of the two-step scenario will require new lattice computations involving dynamical $\Sigma$ fields, which will be the subject of the second paper in this series.

In the present case, we find the following:

(i) There exist regions of model parameter space for which a one-step transition to the Higgs vacuum can be first order. They are shown in Figs. 2, 3, and 4 below. However, without further information, we are unable to assess the strength of the phase transition relevant for baryogenesis.

(ii) For a given value of the physical triplet scalar mass, there is a minimum value of the portal coupling that accommodates a first-order transition. Below this critical value, EWSB occurs via a crossover transition.

(iii) The presence of a first-order transition in this regime is associated with a minimum reduction in the rate for the Higgs boson to decay to two photons.

(iv) These features of the EWPT dynamics are not accessible using perturbative computations.

In the remainder of the paper, we organize our presentation of this analysis as follows. In Sec. II, we formulate and summarize the phenomenology of the $\Sigma$ SM. In Sec. III, we summarize theoretical aspects of dimensional reduction and obtain various DR3EFTs for the case in which $\Sigma$ is a light d.o.f. In Sec. IV, the DR3EFT for the case in which $\Sigma$ is heavy or superheavy is derived, and numeral results are presented. We discuss the implications of our findings in Sec. V. A listing of matching relations among the various DR3EFTs is provided in the Appendixes.

\section{MODEL AND PHENOMENOLOGY}

The $\Sigma \mathrm{SM}$ is formulated by extending the SM with a scalar isotriplet field $\Sigma^{a}$ carrying zero hypercharge. In terms of the SM Higgs isodoublet $H$ and the new isotriplet

$$
H=\left(\begin{array}{c}
\phi^{+} \\
\frac{1}{\sqrt{2}}\left(h+i \phi^{0}\right)
\end{array}\right) \quad \text { and } \quad \Sigma^{a}=\left(\begin{array}{c}
\sigma_{1} \\
\sigma_{2} \\
\sigma_{3}
\end{array}\right) \text {, }
$$


the scalar sector Lagrangian, with the metric signature $(+,-,-,-)$, reads $[24,25]$

$\mathcal{L}=\left(D_{\mu} H\right)^{\dagger}\left(D^{\mu} H\right)+\frac{1}{2}\left(D_{\mu} \Sigma\right)^{a}\left(D^{\mu} \Sigma\right)^{a}-V(H, \Sigma)$,

where the covariant derivatives in terms of the hypercharge and isospin gauge fields $B_{\mu}$ and $W_{\mu}^{a}$ and coupling constants $g^{\prime}$ and $g$ are given by

$$
\begin{aligned}
D_{\mu} H & =\left(\partial_{\mu}+\frac{i}{2} g^{\prime} B_{\mu}+i g \frac{\tau^{a}}{2} W_{\mu}^{a}\right) H \\
\left(D_{\mu} \Sigma\right)^{a} & =\left(\partial_{\mu} \delta^{a c}-g \epsilon^{a b c} W_{\mu}^{b}\right) \Sigma^{c}
\end{aligned}
$$

and the scalar potential is

$$
\begin{aligned}
V(H, \Sigma)= & -\mu^{2} H^{\dagger} H+\lambda\left(H^{\dagger} H\right)^{2}-\frac{1}{2} \mu_{\Sigma}^{2}\left(\Sigma^{a} \Sigma^{a}\right) \\
& +\frac{1}{2} a_{2} H^{\dagger} H \Sigma^{a} \Sigma^{a}+\frac{1}{4} b_{4}\left(\Sigma^{a} \Sigma^{a}\right)^{2} .
\end{aligned}
$$

For simplicity, we have imposed a $Z_{2}$ symmetry under $\Sigma^{a} \rightarrow-\Sigma^{a}$ on the theory that forbids the gauge-invariant cubic portal operator $H^{\dagger} \Sigma^{a} \frac{\tau^{a}}{2} H$. Additionally, we retain only the top-quark Yukawa coupling $y_{t}$ to the SM Higgs doublet, while neglecting all others.

In the potential, we take $\mu^{2}$ positive so that the neutral Higgs field $h$ obtains a nonzero vev at sufficiently low temperature $(T)$, while for high temperature, thermal corrections change the sign of the quadratic operator, leading to symmetry restoration. The sign of the triplet quadratic coefficient, $\mu_{\Sigma}^{2}$, may be either positive or negative. For $\mu_{\Sigma}^{2}>0$, the $T=0$ vacuum exhibits several extrema, including minima along the $h$ and $\sigma_{3}$ directions (for a discussion, see Ref. [25]). Here, we focus on the case in which the absolute $T=0$ minimum lies along the $h$ direction, with a vanishing $\sigma_{3}$ vev. In this vacuum, all three components of $\Sigma^{a}$ are degenerate at leading order, with masses given by

$$
M_{\Sigma}^{2}=-\mu_{\Sigma}^{2}+\frac{1}{2} a_{2} v^{2},
$$

where $v=246 \mathrm{GeV}$ is the zero-temperature tree-level Higgs vev. The physical quanta of charged and neutral scalar fields are $\Sigma^{ \pm}=\left(\sigma_{1} \mp i \sigma_{2}\right) / \sqrt{2}$ and $\Sigma^{0}=\sigma_{3}$. In what follows, we will express our results in terms of the physical mass $M_{\Sigma}$ and the portal coupling $a_{2}$.

The $Z_{2}$ symmetry $\Sigma \rightarrow-\Sigma$ and the absence of a neutral triplet vev implies that $\Sigma^{0}$ is stable. For the range of $M_{\Sigma}$ of interest here (approximately 100-600 GeV), it will contribute a subdominant component of the total dark matter relic density [35]. The corresponding dark matter direct detection constraints on the model parameters can be found in Ref. [36].

Additional constraints may arise from searches for new electroweak multiplets at the LHC. Because of the $Z_{2}$ symmetry, electroweak production of $\Sigma^{ \pm}$and $\Sigma^{0}$ are expected to occur in pairs. Furthermore, electroweak self-energy corrections of $\Sigma$ lead to a small mass splitting between the charged and neutral components by roughly $M_{\Sigma^{ \pm}}-M_{\Sigma^{0}} \approx 160 \mathrm{MeV}$. Consequently, processes involving the production of $\Sigma^{ \pm}$will lead to disappearing charge tracks due to its relatively slow decay to $\Sigma^{0}$ by the emission of a soft pion [24]. Although limits on the existence the charged triplet fermions (e.g., charginos) as a function of the triplet mass and lifetime have been obtained by the ATLAS [37] and CMS [38] collaborations, no significant limits have been placed on the scalar triplet $\Sigma$ due to its much shorter lifetime. Therefore, the LHC results do not yet significantly constrain the model parameter space.

\section{DIMENSIONAL REDUCTION}

In this section, we begin our study of the EWPT in the $\Sigma$ SM by performing a dimensional reduction to an effective three-dimensional theory. We start by providing an overview of dimensional reduction and follow up with its construction as applied to the $\Sigma \mathrm{SM}$. Then, we describe the matching procedure and our power-counting scheme for relating parameters of the various theories. Finally, we state our renormalization scheme to numerically determine the values of input parameters.

\section{A. Overview}

Dimensional reduction is the procedure within the Matsubara imaginary time formalism for constructing an effective three-dimensional theory from the full fourdimensional quantum field theory. The reduction is based on a separation of scales that occurs at high temperature, which we describe below.

Following the nomenclature in Ref. [4], the mass scale associated with the lowest nonvanishing Matsubara frequency $\pi T$ is the "superheavy" scale so that all Matsubara modes apart from the zero mode of bosonic d.o.f. are superheavy. The most prominent dynamical effect of the superheavy modes is to generate thermal masses of order $g T$ for the zero Matsubara modes of scalar fields and time component of the gauge fields. This dynamically generated scale is called the "heavy" scale, which is separated from the superheavy scale in the weak coupling limit. The remaining d.o.f.-spatial components of gauge fieldsare "light" d.o.f.

However, scalar fields of which the bare mass term is negative, such as the $-\mu^{2} H^{\dagger} H$ term of the Higgs isodoublet, will have smaller effective thermal masses due to a cancellation between the bare and the thermally generated ones. At temperatures around the phase transition at which their thermal expectation values are expected to change, the cancellation will be significant to the extent that these scalar fields have effective masses that are far below the 


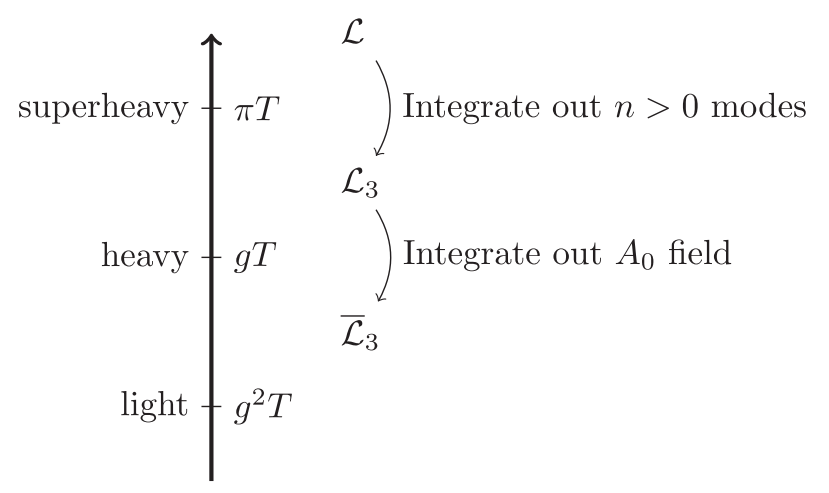

FIG. 1. Separation of scales in equilibrium thermal field theory in the high- $T$ limit and the effective theories associated with each scale. Each effective theory is derived from the one above it by matching Green's functions.

heavy scale. Therefore, the zero Matsubara modes of these scalar fields are also classified as light d.o.f.

This hierarchy of scales in the high- $T$ limit is illustrated in Fig. 1 and motivates us to pass through a series of threedimensional effective field theories, ultimately obtaining a DR3EFT involving just the light d.o.f., which is most readily simulated on the lattice for a nonperturbative study of the EWPT. In the next section, we explain how the effective theories are constructed for the $\Sigma$ SM.

\section{B. Dimensional reduction to the effective theory at the heavy scale}

We begin our construction of the DR3EFTs by first considering the case $\mu_{\Sigma}^{2}>0$ so that the zero Matsubara mode of the real triplet $\Sigma$ is classified as a light d.o.f. This accommodates the possibility for the real triplet to actively participate in the EWPT with a varying thermal expectation value. The case in which $\mu_{\Sigma}^{2}<0$, so that it is classified as heavy or superheavy, will be treated in Sec. IV below.

We start by integrating out the nonzero Matsubara modes (superheavy DOF) to obtain a dimensionally reduced effective theory at the heavy scale involving just the zero Matsubara modes. The most general super-renormalizable Euclidean Lagrangian $\mathcal{L}_{3}$ consistent with the symmetries of the original theory is

$$
\begin{aligned}
\mathcal{L}_{3}= & \frac{1}{4} B_{i j} B_{i j}+\frac{1}{4} W_{i j}^{a} W_{i j}^{a}+\mathcal{L}_{3, \mathrm{gf}}+\left(\vec{D} H^{\dagger}\right) \cdot(\vec{D} H) \\
& +\frac{1}{2}(\vec{D} \Sigma)^{a} \cdot(\vec{D} \Sigma)^{a}+V_{3}(H, \Sigma)+\mathcal{L}_{3, \text { time }}
\end{aligned}
$$

The first few terms resemble the Lagrangian of the underlying four-dimensional theory. The hypercharge and isospin field strength tensors are

$$
\begin{aligned}
B_{i j} & =\nabla_{i} B_{j}-\nabla_{j} B_{i} \\
W_{i j}^{a} & =\nabla_{i} W_{j}^{a}-\nabla_{j} W_{i}^{a}-g_{3} \epsilon^{a b c} W_{i}^{b} W_{j}^{c},
\end{aligned}
$$

the gauge-fixing and SU(2) ghost Lagrangian in the Fermi gauge is

$$
\mathcal{L}_{3, \mathrm{gf}}=\frac{1}{2 \xi}(\vec{\nabla} \cdot \vec{B})^{2}+\frac{1}{2 \xi}\left(\vec{\nabla} \cdot \vec{W}^{a}\right)^{2}+\left(\vec{\nabla} \eta^{a}\right) \cdot(\vec{D} \eta)^{a},
$$

the covariant gradients are

$$
\begin{aligned}
\vec{D} H & =\left(\vec{\nabla}+\frac{i}{2} g_{3}^{\prime} \vec{B}+i g_{3} \frac{\tau^{a}}{2} \vec{W}^{a}\right) H \\
(\vec{D} \Sigma)^{a} & =\left(\vec{\nabla} \delta^{a c}-g_{3} \epsilon^{a b c} \vec{W}^{b}\right) \Sigma^{c},
\end{aligned}
$$

and the scalar potential is

$$
\begin{aligned}
V_{3}(H, \Sigma)= & +\mu_{3}^{2} H^{\dagger} H+\lambda_{3}\left(H^{\dagger} H\right)^{2}+\frac{1}{2} \mu_{\Sigma, 3}^{2}\left(\Sigma^{a} \Sigma^{a}\right) \\
& +\frac{1}{2} a_{2,3} H^{\dagger} H \Sigma^{a} \Sigma^{a}+\frac{1}{4} b_{4,3}\left(\Sigma^{a} \Sigma^{a}\right)^{2} .
\end{aligned}
$$

Additionally, due to the absence of full Lorentz invariance of the theory at finite temperature, additional terms arise in the effective theory involving the time component of gauge fields,

$$
\begin{aligned}
\mathcal{L}_{3, \text { time }}= & \frac{1}{2}\left[\left(\vec{\nabla} W_{0}^{a}\right)^{2}+m_{D}^{2}\left(W_{0}^{a}\right)^{2}\right] \\
& +\frac{1}{2}\left[\left(\vec{\nabla} B_{0}\right)^{2}+m_{D}^{\prime 2} B_{0}^{2}\right]+\frac{1}{2}\left[\left(\vec{\nabla} G_{0}^{A}\right)^{2}+m_{D}^{\prime \prime 2}\left(G_{0}^{A}\right)^{2}\right] \\
& +h_{3} H^{\dagger} H\left(W_{0}^{a}\right)^{2}+h_{3}^{\prime} H^{\dagger} H B_{0}^{2}+h_{3}^{\prime \prime} B_{0} H^{\dagger}\left(W_{0}^{a} \tau^{a}\right) H \\
& +\omega_{3} H^{\dagger} H\left(G_{0}^{A}\right)^{2}+\delta_{3}\left(\Sigma^{a}\right)^{2}\left(W_{0}^{b}\right)^{2}+\delta_{3}^{\prime}\left(\Sigma^{a} W_{0}^{a}\right)^{2} \\
& +\ldots
\end{aligned}
$$

We have omitted terms involving self-interactions of time components of gauge fields that do not contribute to the light scale DR3EFT at the level of precision at which we work mentioned below. Formulas connecting the coupling constants and the normalization of the fields in $\mathcal{L}_{3}$ to the couplings and zero Matsubara modes of the full fourdimensional theory in $\mathcal{L}$ are obtained by matching, which we explain in more detail in Sec. III D and are listed in Appendix A 2.

\section{Reduction to the theory at the light scale}

As explained above, the effect of integrating out the nonzero Matsubara modes at the superheavy scale is to induce thermal masses of scalar fields and the time component of gauge fields of order $g T$, which in the weak coupling limit are separated from the superheavy scale but in the high-temperature limit are separated from the light scale. Continuing with our assumption that $\Sigma$ is light, the only d.o.f. at the heavy scale that need to be integrated out to obtain an effective theory at the light scale are the time component of gauge fields $B_{0}, W_{0}^{a}$, and $G_{0}^{A}$. 
The most general super-renormalizable effective Lagrangian involving the light d.o.f. is

$$
\begin{aligned}
\overline{\mathcal{L}}_{3}= & \frac{1}{4} B_{i j} B_{i j}+\frac{1}{4} W_{i j}^{a} W_{i j}^{a}+\overline{\mathcal{L}}_{3, \mathrm{gf}}+\left(\vec{D} H^{\dagger}\right) \cdot(\vec{D} H) \\
& +\frac{1}{2}(\vec{D} \Sigma)^{a} \cdot(\vec{D} \Sigma)^{a}+\bar{V}_{3}(H, \Sigma),
\end{aligned}
$$

with the same abbreviations listed in (7)-(10), but with new couplings that we distinguish with a bar: $\bar{g}_{3}, \bar{g}_{3}^{\prime}, \bar{\lambda}_{3}, \bar{a}_{2,3}$, etc. The form of the effective Lagrangian at the light scale is identical to that at the heavy scale in (6), but without $\mathcal{L}_{3, \text { time }}$. The relations connecting the coupling constants at the heavy scale and the light scale are derived by matching and are listed in Appendix A 3. This effective theory will be analyzed nonperturbatively on the lattice in Part II of this study.

\section{Matching of the parameters}

In this subsection, we explain how the field normalizations and coupling constants between sets of EFTs are derived. Additionally, we specify our power-counting scheme and the level of precision at which we derive these matching relations. For details of the matching procedure, see also Refs. [4,39].

We adopt a power-counting scheme similar to that of Ref. [4], wherein the quartic couplings scale as the square of the $\mathrm{SU}(2)$ gauge coupling constant

$$
\lambda, a_{2}, b_{4} \sim g^{2},
$$

while the top-quark Yukawa coupling and the remaining gauge coupling constants scale linearly,

$$
y_{t}, g^{\prime}, g_{s} \sim g .
$$

Additionally, as explained in Sec. III A above, under the assumption that both the Higgs doublet $H$ and the real triplet $\Sigma$ are light, the corresponding negative mass parameters scale as the square of the gauge coupling constant,

$$
\mu^{2}, \mu_{\Sigma}^{2} \sim g^{2} T^{2},
$$

near the electroweak phase transition.

We perform the dimensional reduction perturbatively and use dimensional regularization to regulate divergent sum integrals. We ultimately aim to obtain the DR3EFT at the light scale in (12) to order $O\left(g^{4}\right)$. This requires the evaluation of self-energy functions through two loops to match mass parameters $\mu^{2}$ and $\mu_{\Sigma}^{2}$ and the remaining Green's functions through one loop to match the coupling constants. To illustrate how the matching relations for fields and couplings between the EFTs are derived, we summarize the procedure, using the portal couplings $a_{2,3}$ and $\bar{a}_{2,3}$ as a representative example.

The formula for $a_{2,3}$ listed in (A23) is determined by requiring that the four-point Green's function $\left\langle H^{\dagger} H \Sigma^{a} \Sigma^{a}\right\rangle$ in both the four-dimensional theory and the heavy-scale three-dimensional (3D) theory match at the matching scale $\Lambda$. This is possible provided the fields in the 3D theory are canonically normalized. Canonical normalization is achieved by comparing the two-point Green's function in the two theories. For a generic bosonic field $\phi$, this relationship reads

$$
\phi_{3 \mathrm{~d}}^{2}=\frac{1}{T}\left[1+\hat{\Pi}_{\phi}^{\prime}(0,0)\right] \phi^{2},
$$

where $\hat{\Pi}_{\phi}\left(\omega^{2}, \vec{p}^{2}\right)$ is the fully renormalized self-energy function of the Matsubara frequency $\omega$ and spatial momentum $\vec{p}$ and the prime denotes a derivative with respect to $\vec{p}^{2}$. The explicit factor of $1 / T$ accounts for absorbing a similar factor in front of the 3D effective Lagrangian. To ultimately obtain an $\mathcal{O}\left(g^{4}\right)$ accuracy in the matching relations, $\hat{\Pi}_{\phi}^{\prime}$ in (16) needs to be known only to one-loop order. Additionally, only contributions from the $n \neq 0$ Matsubara modes should be included.

The portal coupling $a_{2,3}$ can be then be determined by comparing the corresponding tree-level vertex in the DR3EFT against the one in four dimensions calculated to $O\left(g^{4}\right)$. The $3 \mathrm{D}$ vertex reads

$$
-a_{2,3} T\left(H^{\dagger} H \Sigma^{a} \Sigma^{a}\right)_{3 \mathrm{~d}},
$$

where the factor of $T$ follows from the rescaling of the $3 \mathrm{D}$ fields. The corresponding vertex in the four-dimensional theory is

$$
\left(-a_{2}-\hat{\Gamma}(0)\right)\left(H^{\dagger} H \Sigma^{a} \Sigma^{a}\right)_{4 \mathrm{~d}}
$$

where $\hat{\Gamma}(0)$ is the connected (fully renormalized) one-loop $H^{\dagger} H \Sigma^{a} \Sigma^{a}$ vertex function at zero external momentum and excludes the zero Matsubara modes. By matching (17) and (18) and accounting for the difference in the field normalization in (16), we obtain the desired matching formula for the portal coupling:

$$
a_{2,3}=T\left[a_{2}-a_{2}\left(\hat{\Pi}_{H}^{\prime}(0)+\hat{\Pi}_{\Sigma}^{\prime}(0)\right)+\hat{\Gamma}(0)\right] .
$$

All other matching relations between the superheavy and heavy scales listed in Appendix A 2 are derived in a similar way, using the table of integrals found in Ref. [4]. To minimize logarithms, and to eliminate $\ln (4 \pi)-\gamma_{E}$ associated with dimensional regularization, we choose the matching scale to be $\Lambda=4 \pi T / e^{\gamma_{E}}$.

To obtain the portal coupling $\bar{a}_{2,3}$ at the light scale, in which the time component of gauge fields $B_{0}, W_{0}^{a}$, and $G_{0}^{A}$ are integrated out, an analogous procedure is followed. Field and mass parameters are again related by comparing self-energy functions. However, there is no change in normalization of the scalar fields in the two theories as there are no contributions giving momentum dependence. This leads to the simpler matching relation 


$$
\bar{a}_{2,3}=a_{2,3}+\hat{\Gamma}_{3}(0),
$$

where $\hat{\Gamma}_{3}(0)$ is the contribution from the $B_{0}, W_{0}^{a}$, and $G_{0}^{A}$ fields to the $H^{\dagger} H \Sigma^{a} \Sigma^{a}$ connected Green's function in the "high" scale DR3EFT.

Because of the super-renormalizability of the light scale DR3EFT, only the self-energy functions of the Higgs doublet $H$ and the triplet $\Sigma$ are UV divergent. Consequently, the matching scale $\Lambda_{0}$ formally appears in the matching relations for $\bar{\mu}_{3}^{2}$ and $\bar{\mu}_{\Sigma, 3}^{2}$. Moreover, the anomalous dimensions of the mass parameters terminate at two-loop order, and the associated renormalization group equations can be solved exactly and take the form

$$
\mu_{3}^{2}(\Lambda)=f_{3} \ln \frac{\Lambda_{0}}{\Lambda_{3 \mathrm{~d}}}
$$

where $f_{3}$ is a function of the $3 \mathrm{D}$ couplings (corresponding to the mass counterterm), $\Lambda_{3 \mathrm{~d}}$ is the renormalization group scale of the $3 \mathrm{D}$ theory, and $\Lambda_{0}$ is a mass scale that is determined by the matching procedure. By a direct calculation of two-loop logarithms in four dimensions, one may identify the constant $\Lambda_{0}$ and replace the two-loop logarithms by the more accurate expression in Eq. (21).

\section{E. Renormalization and the numerical determination of parameters}

For a numerical study of the phase diagram in this model, it remains to fix the input parameters of the underlying model at the superheavy scale. The theory depends on five parameters of the SM, $\mu^{2}, \lambda, g^{\prime}, g$, and $y_{t}$, in addition to three parameters from the extended sector, $\mu_{\Sigma}^{2}, a_{2}$, and $b_{4}$. We determine their values in the $\overline{\mathrm{MS}}$ scheme by relating them to measured observables.

We choose to fix $\mu^{2}, \lambda, g^{\prime}$, and $g$ by relating them to the fine structure constant $\hat{\alpha}\left(M_{Z}^{2}\right)$ and the pole masses $M_{W}$, $M_{Z}$, and $M_{H}$, at the scale $\Lambda=M_{Z}$. Although the Fermi constant $G_{F}$ is conventionally used in place of $M_{W}$ for a more precise determination, at the level of precision at which we are working, we choose to work with $M_{W}$ for clarity. In terms of the Higgs self-energy function $\Sigma_{H}$ and the transverse polarization functions of the gauge bosons $\Pi_{W}$ and $\Pi_{Z}$, the one-loop relations are

$$
\begin{gathered}
\mu_{H}^{2}=\frac{M_{H}^{2}}{2}\left(1-\frac{\Sigma_{H}\left(M_{H}^{2}\right)}{M_{H}^{2}}\right) \\
\lambda=\frac{\pi \hat{\alpha} M_{H}^{2} M_{Z}^{2}}{2 M_{W}^{2}\left(M_{Z}^{2}-M_{W}^{2}\right)}\left[1-\frac{\Sigma_{H}\left(M_{H}^{2}\right)}{M_{H}^{2}}-\frac{\Pi_{Z}\left(M_{Z}^{2}\right)}{M_{Z}^{2}}\right. \\
\left.+\frac{\Pi_{W}\left(M_{W}^{2}\right)}{M_{W}^{2}}+\frac{\Pi_{Z}\left(M_{Z}^{2}\right)-\Pi_{W}\left(M_{W}^{2}\right)}{M_{Z}^{2}-M_{W}^{2}}\right] \\
g^{\prime 2}=\frac{4 \pi \hat{\alpha} M_{Z}^{2}}{M_{W}^{2}}\left[1-\frac{\Pi_{Z}\left(M_{Z}^{2}\right)}{M_{Z}^{2}}+\frac{\Pi_{W}\left(M_{W}^{2}\right)}{M_{W}^{2}}\right]
\end{gathered}
$$

$$
\begin{aligned}
g^{2}= & \frac{4 \pi \hat{\alpha} M_{Z}^{2}}{M_{Z}^{2}-M_{W}^{2}}\left[1-\frac{\Pi_{Z}\left(M_{Z}^{2}\right)}{M_{Z}^{2}}\right. \\
& \left.+\frac{\Pi_{Z}\left(M_{Z}^{2}\right)-\Pi_{W}\left(M_{W}^{2}\right)}{M_{Z}^{2}-M_{W}^{2}}\right] .
\end{aligned}
$$

The relationship for the top-quark Yukawa coupling additionally depends on its self-energy function, parametrized in terms of invariant functions as

$$
-i \Sigma(\not p)=-i\left(\not p A\left(p^{2}\right)+M_{t} B\left(p^{2}\right)\right) .
$$

At one-loop order, the relationship is

$$
\begin{aligned}
y_{t}^{2}= & 2 \pi \hat{\alpha} \frac{M_{Z}^{2} M_{t}^{2}}{M_{W}^{2}\left(M_{Z}^{2}-M_{W}^{2}\right)}\left[1-\frac{\Pi_{Z}\left(M_{Z}^{2}\right)}{M_{Z}^{2}}+\frac{\Pi_{W}\left(M_{W}^{2}\right)}{M_{W}^{2}}\right. \\
& \left.+\frac{\Pi_{Z}\left(M_{Z}^{2}\right)-\Pi_{W}\left(M_{W}^{2}\right)}{M_{Z}^{2}-M_{W}^{2}}-2\left(A\left(M_{t}^{2}\right)+B\left(M_{t}^{2}\right)\right)\right],
\end{aligned}
$$

which we use to fix the Yukawa coupling at the scale $\Lambda=M_{t}$.

Finally, among the three parameters of the extended sector, we only choose to express the mass parameter of the real triplet $\mu_{\Sigma}^{2}$ in terms of the physical pole mass of the electrically neutral triplet $\Sigma^{0}$ at the scale $\Lambda=M_{\Sigma}$. In terms of the neutral triplet self-energy function $\Sigma_{\Sigma}$, the one-loop relationship is given by

$$
\begin{aligned}
\mu_{\Sigma}^{2}= & -M_{\Sigma}^{2}+\Sigma_{\Sigma}\left(M_{\Sigma}^{2}\right)+\frac{a_{2} M_{W}^{2}}{2 \pi \hat{\alpha}}\left(1-\frac{M_{W}^{2}}{M_{Z}^{2}}\right) \\
& \times\left[1-\frac{\Pi_{W}\left(M_{W}^{2}\right)}{M_{W}^{2}}-\frac{\Pi_{W}\left(M_{W}^{2}\right) / M_{W}^{2}-\Pi_{Z}\left(M_{Z}^{2}\right) / M_{Z}^{2}}{1-M_{Z}^{2} / M_{W}^{2}}\right] .
\end{aligned}
$$

Since no meaningful measurements have been made to fix the remaining parameters $a_{2}$ and $b_{4}$, in what follows, we will present our results directly in terms of their $\overline{\mathrm{MS}}$ values at the scale $\Lambda=M_{Z}$.

Having determined the values of renormalized parameters at their chosen scales, we solve the one-loop renormalization group equations

$$
\begin{gathered}
\Lambda \frac{d g^{2}}{d \Lambda}=-\frac{g^{4}}{8 \pi^{2}}\left(\frac{22}{3}-\frac{N_{d}+2 N_{t}}{6}-\frac{4}{3} N_{f}\right), \\
\Lambda \frac{d g^{\prime 2}}{d \Lambda}=\frac{g^{\prime 4}}{8 \pi^{2}}\left(\frac{N_{d}}{6}+\frac{20}{9} N_{f}\right), \\
\Lambda \frac{d y_{t}^{2}}{d \Lambda}=\frac{y_{t}^{2}}{8 \pi^{2}}\left(\frac{9}{2} y_{t}^{2}-\frac{9}{4} g^{2}-\frac{17}{12} g^{\prime 2}-8 g_{s}^{2}\right),
\end{gathered}
$$




$$
\Lambda \frac{d \mu^{2}}{d \Lambda}=\frac{1}{16 \pi^{2}}\left(-3 \mu^{2}\left(\frac{3}{2} g^{2}+\frac{1}{2} g^{2}-2 y_{t}^{2}-4 \lambda\right)+3 \mu_{\Sigma}^{2} a_{2}\right)
$$

$$
\begin{gathered}
\Lambda \frac{d \mu_{\Sigma}^{2}}{d \Lambda}=\frac{1}{16 \pi^{2}} 2\left(2 a_{2} \mu^{2}-6 g^{2} \mu_{\Sigma}^{2}+5 b_{4} \mu_{\Sigma}^{2}\right) \\
\Lambda \frac{d \lambda}{d \Lambda}=\frac{1}{16 \pi^{2}} \frac{1}{2}\left(48 \lambda_{1}^{2}+3 a_{2}^{2}+\frac{3}{4}\left(3 g^{4}+g^{\prime 4}+2 g^{2} g^{\prime 2}\right)\right. \\
\left.-12 y_{t}^{4}-6 \lambda\left(3 g^{2}+g^{\prime 2}-4 y_{t}^{2}\right)\right) \\
\Lambda \frac{d a_{2}}{d \Lambda}=\frac{1}{16 \pi^{2}} 2\left(a _ { 2 } \left(-\frac{33}{4} g^{2}-\frac{3}{4} g^{2}+3 y_{t}^{2}\right.\right. \\
\Lambda \frac{d b_{4}}{d \Lambda}=\frac{1}{16 \pi^{2}} 2\left(-12 b_{4} g^{2}+a_{2}^{2}+11 b_{4}^{2}+6 g^{4}\right)
\end{gathered}
$$

to obtain their values at the matching scale $\Lambda=4 \pi T / e^{\gamma_{E}}$. By allowing the parameters in the tree-level Lagrangian to vary with the renormalization scale, we observe that our results exhibit reduced sensitivity to the matching scale $\Lambda$.

Having derived the DR3EFT at the light scale (12) and established a renormalization scheme to fix the input parameters, the next step is to perform a numerical study of this theory on the lattice. We postpone the lattice formulation of this theory, together with a comparison of numerical results with perturbation theory, to Part II of this series. Instead, in the next section, we turn to the case in which the real triplet $\Sigma$ is either heavy or superheavy, for which we can use existing lattice results to study the EWPT.

\section{HEAVY AND SUPERHEAVY TRIPLETS}

In the case $\mu_{\Sigma}^{2}<0$, the real triplet d.o.f. $\Sigma$ are either at the heavy or superheavy scales. This requires that they are integrated out in the first or second step of dimensional reduction and is therefore absent from the DR3EFT at the light scale. Although this assumption precludes the possibility of $\Sigma$ changing its thermal expectation value during the EWPT, the resulting DR3EFT is of the same form as that obtained from the minimal SM,

$$
\bar{V}_{3}(H)=\bar{\mu}_{3}^{2} H^{\dagger} H+\bar{\lambda}_{3}\left(H^{\dagger} H\right)^{2}
$$

but here the influence of the heavy or superheavy $\Sigma$ is encoded in the matching relations listed in Appendixes A 4 and B, respectively. Since the thermodynamics of the EWPT of this theory has previously been studied on the lattice [4], we may readily apply the results in this case to study the EWPT in the $\Sigma$ SM.
Properties of the EWPT on the lattice are characterized by two temperature-dependent dimensionless parameters:

$$
x=\frac{\bar{\lambda}_{3}}{\bar{g}_{3}^{2}}, \quad y=\frac{\bar{\mu}_{3}^{2}}{\bar{g}_{3}^{4}} .
$$

The results of the simulations are as follows. The critical temperature occurs near where the $y$ parameter changes sign; when $x$ is sufficiently small but positive $0<x \lesssim 0.11$, the EWPT is first order [8]. At $x \approx 0.11$, the system exhibits a second-order EWPT, and for larger values of $x$, the transition is a crossover. We note that the upper bound on $x$ has been obtained using 3D lattice results for the $\mathrm{SU}(2)$ plus Higgs theory and allowing for an approximately $10 \%$ correction from neglected $\mathrm{U}(1)_{Y}$ contributions. In Sec. IV $B$, we will present our results based on the numerical analysis for the case in which $\Sigma$ is a heavy d.o.f. We make a comparison with the superheavy case in Appendix B.

\section{A. Validity of dimensional reduction}

Following Ref. [4], we can check the validity of the dimensional reduction by estimating the impact of the higher-dimensional operators that have been dropped from the light scale DR3EFT on the vevs of the scalars in the effective theory.

The lowest-dimension operators omitted from the heavy and light scales are the (marginal) dimension-3 operators $c_{3}\left(H^{\dagger} H\right)_{3 \mathrm{~d}}^{3}$ and $\bar{c}_{3}\left(H^{\dagger} H\right)_{3 \mathrm{~d}}^{3}$, respectively. Upon integrating out the superheavy scale, the coefficient of the operator at the heavy scale is

$$
\begin{aligned}
c_{3}= & \frac{\zeta(3)}{16384 \pi^{4}}\left(3 g^{6}+g^{6}+3 g^{2} g^{2}\left(g^{2}+g^{2}\right)+640 \lambda^{3}\right. \\
& \left.-224 y_{t}^{6}+8 a_{2}^{3}\right) .
\end{aligned}
$$

The top-quark contribution dominates over other SM contributions. The dominant correction $\bar{c}_{3}$ in the $\Sigma \mathrm{SM}$ comes not from the superheavy scale but from the second step of DR when the heavy triplet is integrated out. The total dimension-3 coefficient can be written as

$$
\bar{c}_{3}=c_{3}+c_{3}^{\text {heavy }}
$$

and the $\Sigma \mathrm{SM}$ contribution to $\Lambda_{6}^{\text {heavy }}$ is

$$
c_{3}^{\text {heavy }}(\Sigma)=\frac{1}{512 \pi}\left(\frac{a_{2,3}}{\mu_{\Sigma, 3}}\right)^{3} \text {. }
$$

Note that the time component of gauge fields has a subdominant effect when integrating out the heavy scale [4].

The top-quark contribution

$$
c_{3}(\text { top })=-\frac{7 \zeta(3)}{512 \pi^{4}} y_{t}^{6}
$$


shifts the position of the Higgs vev by about $1 \%$ in the pure SM [4]. We can estimate the effect of the dimension-3 $\left(H^{\dagger} H\right)^{3}$ operator by comparing the magnitude of the dominant $\Sigma$ SM contribution to that of the top quark. If the ratio

$$
\Delta_{6} \equiv\left|\frac{c_{3}^{\text {heavy }}(\Sigma)}{c_{3} \text { (top) }}\right|
$$

becomes large, the validity of the DR3EFT is compromised.

\section{B. Results for one-step transition with superheavy and heavy triplets}

With the foregoing DR3EFT setup for the heavy $\Sigma$ and matching conditions in hand, we map out the phase diagram for the theory in the $\left(M_{\Sigma}, a_{2}\right)$ plane by scanning over the parameters of the potential, determining the values of $x$ and $y$ in (37) and identifying the region for a first-order EWPT as obtained in the study of Ref. [8]. We have performed this scan over the $\left(M_{\Sigma}, a_{2}\right)$-parameter space assuming a uniform distribution of the parameters. The triplet mass was varied from 100 to $600 \mathrm{GeV}$ at intervals of $5 \mathrm{GeV}$ and portal coupling $a_{2}$ from 0 to 4 at intervals of 0.05 . We then omit all the points in which the triplet mass parameter squared is positive according to tree-level relation.

For each point, we scan the temperature from 80 to $200 \mathrm{GeV}$ at intervals of $20 \mathrm{GeV}$ and find the critical temperature $T_{c}$ by interpolation from the condition that $y=0$. To obtain the phase diagram, we determine the value of $x\left(T_{c}\right)$ at each point in the parameter space. For purposes of visualization, we perform a linear interpolation to obtain contours of constant $x$. We present our results with fixed $b_{4}=0.75$ and verified that the values of $x$ and $y$ at the critical temperature are not strongly sensitive to it.

The results are displayed in Figs. 2, 3, and 4. In each case, we indicate regions where the EWSB transition from the high- $T$ symmetric phase is a one-step crossover or first order transition, corresponding to the light blue and light green regions, respectively. The dark green regions correspond to choices of the parameters for which the validity of the DR3EFT breaks down. The gray regions, above and to the left of the line $\mu_{\Sigma}^{2}=0$, indicate regions of parameter space for which the $\Sigma$ is light and where its inclusion in lattice simulations is required. Consequently, we make no statement about the phase diagram for this region. We anticipate, however, that the two-step transition analyzed perturbatively in Ref. [25] will emerge in this region from the future lattice study of the gray region.

A key feature of each plot is the existence of a choice of parameters giving a first-order or a crossover transition as well as the phase boundary between the two situations. We emphasize that one cannot identify the existence of the cross over region and the boundary with the first-order region from a purely perturbative analysis. The results given here thus underscore the importance of going beyond

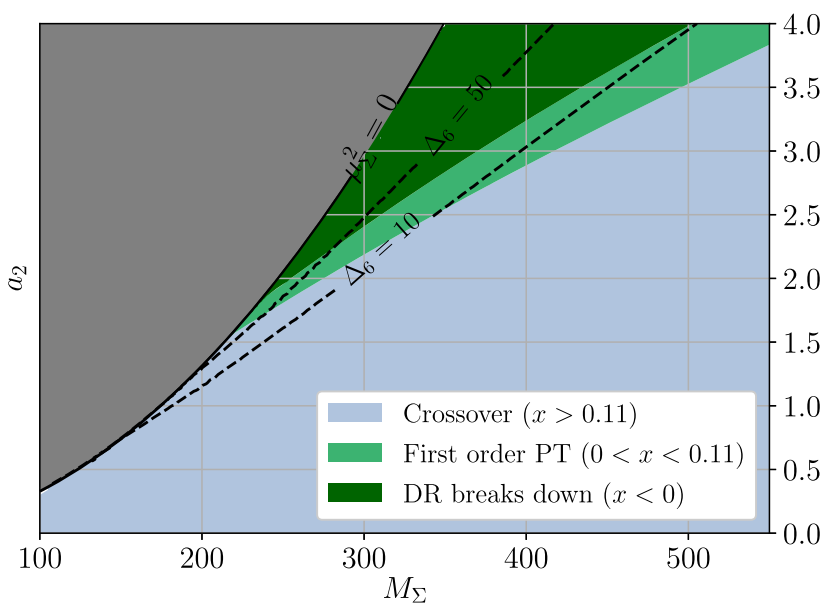

FIG. 2. $\Sigma$ SM phase diagram for a heavy triplet as a function of the triplet mass $M_{\Sigma}$ and the portal coupling $a_{2}$. Light blue and light green regions correspond to a one-step crossover and firstorder EWSB transition to the Higgs vacuum, respectively, starting from the electroweak symmetric phase at high $T$. In the dark green region, assumptions of dimensional reduction (DR) no longer hold. The gray region corresponds to $\mu_{\Sigma}^{2}>0$, where the real triplet is expected to participate in EWPT and must be classified as a light d.o.f. Therefore, this area of parameter space needs inclusion of its dynamics in the Monte Carlo simulations, so no statement about the phase structure is made here. The dotted lines indicate contours of constant $\Delta_{6}$ defined in (41).

perturbation theory in order to obtain a physically complete and quantitatively realistic picture of the phase structure of the theory.

Going beyond this primary point, each of Figs. 2, 3, and 4 contain a set of dashed curves that highlight various theoretical and phenomenological considerations. The dashed curves in Fig. 2 give contours of constant $\Delta_{6}$, defined



FIG. 3. Same as in Fig. 2, but showing the relative change $\delta$ in the partial width $\Gamma(h \rightarrow \gamma \gamma)$, defined in (42). Dashed lines indicate contours of constant $\delta$ for regions of the parameter space relevant to this analysis. 




FIG. 4. Same is in Fig. 2, but showing values of the critical temperature. Dashed lines show contours of constant $T_{c}$ in the vicinity of the first-order one-step transition.

in Eq. (41). Recall that $\Delta_{6}$ characterizes the relative magnitudes of $\Sigma$ and top-quark contributions to the coefficient of the higher-dimensional $\left(H^{\dagger} H\right)^{3}$ operator in the potential. A rough indication of the importance of this operator on the quantities relevant to the phase transition was obtained in Ref. [4], in which it was shown that the presence of $c_{3}$ (top) leads to a $1 \%$ shift in the value of the Higgs vev. We would thus expect the relative impact of $c_{3}^{\text {heavy }}(\Sigma)$ to scale linearly with the ratio $\Delta_{6}$. For sufficiently large $a_{2}$ and light $M_{\Sigma}$, one would thus expect corrections of greater than approximately $10 \%$ in the value of the Higgs vev associated with the tripletinduced higher-dimension operators. The value of the Higgs vev itself is, of course, not directly relevant to the boundaries of the phase diagram, the critical temperature, etc., but it does provide one way to assess the quantitative impact of theoretical uncertainties. We defer a more complete determination of the corrections from higher-dimensional operators on the phase transition properties to future work and take the contours of constant $\Delta_{6}$ as rough indications of the accuracy of our present DR3EFT treatment.

In Fig. 3, we illustrate the implications of this study for measurements of Higgs boson couplings. Of particular interest is the rate for the decay to two photons, $\Gamma(h \rightarrow \gamma \gamma)$. As discussed in detail in Refs. [24,25], loops involving the charged components of the triplet will contribute to the diphoton decay rate, shifting its value from the SM prediction as a function of $\left(M_{\Sigma}, a_{2}\right)$. Defining the relative shift

$$
\delta=\frac{\Gamma^{\Sigma \mathrm{SM}}(h \rightarrow \gamma \gamma)-\Gamma^{\mathrm{SM}}(h \rightarrow \gamma \gamma)}{\Gamma^{\mathrm{SM}}(h \rightarrow \gamma \gamma)},
$$

we plot in Fig. 3 contours of constant $\delta$ in the vicinity of the first-order transition region and the boundary with the crossover region. Note that in call cases, $\delta<0$. We emphasize that each point along the boundary between the first-order and crossover regions corresponds to a minimum value of $|\delta|$. This feature would allow one to exploit a measurement of $\Gamma(h \rightarrow \gamma \gamma)$ (or the corresponding branching ratio) to probe the nature of the transition. For fixed $M_{\Sigma}$, for example, a sufficiently large and negative deviation of the diphoton rate would indicate the existence of a firstorder transition, whereas a smaller magnitude or positive value for $\delta$ would imply a crossover transition.

A separate experimental study would be required identifying $M_{\Sigma}$. Under the assumptions of the study here, wherein $\Sigma^{0}$ obtains no vev, such a study could include the search for disappearing charge tracks, as discussed in Ref. [24]. One expects the high-luminosity phase of the Large Hadron Collider to enable a determination of the diphoton rate with approximately 5\% $-10 \%$ precision [40], potentially allowing one to probe the lower $M_{\Sigma}$ region of the green regions of Figs. 2-4. A conclusive test of the nature of the transition in the region of parameter space considered here may require a future $e^{+} e^{-}$and/or $p p$ collider that is able to achieve a better than 5\% determination of $\Gamma(h \rightarrow \gamma \gamma)$ and a separate determination of $M_{\Sigma}$. One may also anticipate other loop-induced Higgs property deviations, ${ }^{3}$ such as the rate for associated production $e^{+} e^{-} \rightarrow Z^{*} \rightarrow Z h$.

Figure 4 contains contours of constant $T_{c}$ in the vicinity of the first-order transition region. Knowledge of the critical temperature is interesting in its own right as well as for assessing the validity of the DR3EFT. We observe that for the parameter choices in the first-order region the physical triplet mass $M_{\Sigma}$ is greater than $T_{c}$, validating our treatment of the triplet as a heavy d.o.f. Only for sufficiently large $M_{\Sigma}$ would the superheavy triplet DR3EFT be justified, giving a posteriori justification for concentrating on the heavy rather than the superheavy case.

Looking to the future, knowledge of $T_{c}$ will be important for assessing the strength of the phase transition in the light green region. We emphasize that our present study provides no information about the quantities that characterize the strength of the transition, such as the broken phase sphaleron rate relevant to electroweak baryogenesis or the latent heat and effective action relevant to the dynamics of gravitational radiation generated during a first-order transition [41]. In principle, one could estimate the broken phase sphaleron rate using a combination of analytic and numerical methods (see Ref. [42] and references therein), a task that requires knowledge of the bubble nucleation temperature that is often reasonably approximated by $T_{c}$ but that goes beyond the scope of the present study. A more robust determination of the sphaleron rate would require a nonperturbative study. Similar comments apply to the thermodynamic quantities relevant to gravitational wave generation. We defer an in-depth analysis of these issues to future work.

\footnotetext{
${ }^{3}$ We thank Lian-Tao Wang for raising this possibility.
} 


\section{DISCUSSION}

In this paper, we have initiated a nonperturbative study of the electroweak phase transition in the $\Sigma$ SM. We have performed a finite-temperature dimensional reduction in this model and derived a set of effective three-dimensional theories that can be studied by lattice simulations. We have immediately applied these effective theories in the case in which the triplet is assumed to be sufficiently heavy that it may be integrated out, leading to effective 3D theory of same form as in the SM, and existing lattice results of Ref. [8] can be applied. We have found that there exist regions for which a one-step transition to the EWSB vacuum can be of first order. In addition, for a given value of triplet mass, there is a minimum value of the portal coupling that can accommodate a first-order transition. Below this critical value, the EWPT is a smooth crossover, as in the minimal SM. We emphasize that, in order to reach this conclusion, a nonperturbative treatment is crucial, since perturbative analyses cannot identify the existence of the crossover region. Furthermore, we have shown that the presence of a first-order transition is associated with a lower bound on the $h \rightarrow \gamma \gamma$ partial width. This bound would potentially allow one to probe regions of the parameter space, allowing a first-order EWPT with the high-luminosity phase of the Large Hadron Collider or with a future $e^{+} e$ and/or $p p$ collider.

We emphasize that our study of EWPT as it stands is limited to providing the critical temperature and character of the EWPT (first order, second order, or crossover). Without external information, nonequilibrium thermodynamic properties, such as latent heat or bubble nucleation rate, relevant for the gravitational wave generation, or the broken phase sphaleron rate relevant to electroweak baryogenesis, cannot be inferred.

The existence of a crossover transition and the presence of a critical boundary between regions of crossover and first-order transition can be revealed only in nonperturbative analysis. Despite this, frequently used perturbative studies may potentially provide a reasonable qualitative, if not quantitative, agreement with lattice on other features of the EWPT. To test the reliability of the perturbative approach, in Part II, we will perform a systematic comparative analysis of the performance of perturbation theory to extract thermodynamic quantities, which would allow us to set a definite benchmark for the accuracy of the perturbation theory.

\section{ACKNOWLEDGMENTS}

T. T. has been supported by the Vilho, Yrjö and Kalle Väisälä Foundation. L. N. and T. T. have been supported by the Academy of Finland Grant No. 273545 as well as by the European Research Council Grant No. 725369. L. N. was also supported by the Academy of Finland Grant No. 308791. D. J. W. (ORCID ID 0000-0001-6986-0517) was supported by Academy of Finland Grant No. 286769 and by the Research Funds of the University of Helsinki. H. H. P. and M. J. R. M. were supported in part under U.S. Department of Energy Contract No. DE-SC0011095. The authors would like to thank Keijo Kajantie, Jonathan Kozaczuk, Mikko Laine, Kari Rummukainen, and Aleksi Vuorinen for discussions.

\section{APPENDIX A: MATCHING RELATIONS- $-\Sigma$ HEAVY OR LIGHT}

In this Appendix, we list the matching relations of the normalization of fields and coupling constants between the three-dimensional effective theory at the heavy scale $\mathcal{L}_{3}$ in (6) and the full four-dimensional theory at the superheavy scale $\mathcal{L}$. The relations are valid for case in which the real triplet d.o.f. $\Sigma^{a}$ are classified as either heavy or light. Matching relations for the case in which $\Sigma^{a}$ is superheavy are provided in the next section.

In the following expressions, we introduce $N_{d}=1$, $N_{t}=1$, and $N_{f}=3$ to identify contributions from the SM Higgs doublet, the real triplet $\Sigma$, and fermions. Additionally, we make the following abbreviations arising from the evaluation of one- and two-loop integrals in dimensional regularization:

$$
\begin{gathered}
L_{b}=\ln \left(\frac{\Lambda^{2}}{T^{2}}\right)-2[\ln (4 \pi)-\gamma], \\
L_{f}=L_{b}+4 \ln 2, \\
c=\frac{1}{2}\left(\ln \left(\frac{8 \pi}{9}\right)+\frac{\zeta^{\prime}(2)}{\zeta(2)}-2 \gamma_{E}\right) .
\end{gathered}
$$

\section{Normalization of fields}

Here, we collect normalizations between the four- and three-dimensional fields in the Landau gauge $\xi=0$. Field normalizations of $B_{0}, \vec{B}$, and $H$ are not affected by scalar triplet $\Sigma$ and are therefore same as in the SM:

$$
\begin{aligned}
W_{3 \mathrm{~d}, 0}^{2}= & \frac{W_{4 \mathrm{~d}, 0}^{2}}{T}\left[1+\frac{g^{2}}{(4 \pi)^{2}}\left(\frac{N_{d}+2 N_{t}-26}{6} L_{b}\right.\right. \\
+ & \left.\left.\frac{1}{3}\left(8+N_{d}+2 N_{t}\right)+\frac{4 N_{f}}{3}\left(L_{f}-1\right)\right)\right], \\
\vec{W}_{3 \mathrm{~d}}^{2}= & \frac{\vec{W}_{4 \mathrm{~d}}^{2}}{T}\left[1+\frac{g^{2}}{(4 \pi)^{2}}\left(\frac{N_{d}+2 N_{t}-26}{6} L_{b}\right.\right. \\
& \left.\left.-\frac{2}{3}+\frac{4 N_{f}}{3} L_{f}\right)\right], \\
B_{3 \mathrm{~d}, 0}^{2}= & \frac{B_{4 \mathrm{~d}, 0}^{2}}{T}\left[1+\frac{g^{\prime 2}}{(4 \pi)^{2}}\left(N_{d}\left(\frac{L_{b}}{6}+\frac{1}{3}\right)\right.\right. \\
& \left.\left.+\frac{20 N_{f}}{9}\left(L_{f}-1\right)\right)\right]
\end{aligned}
$$




$$
\begin{array}{r}
\vec{B}_{3 \mathrm{~d}}^{2}=\frac{\vec{B}_{4 \mathrm{~d}}^{2}}{T}\left[1+\frac{g^{\prime 2}}{(4 \pi)^{2}}\left(N_{d} \frac{L_{b}}{6}+\frac{20 N_{f}}{9} L_{f}\right)\right] . \\
\left(H^{\dagger} H\right)_{3 \mathrm{~d}}=\frac{\left(H^{\dagger} H\right)_{4 \mathrm{~d}}}{T}\left[1-\frac{1}{(4 \pi)^{2}}\left(\frac{3}{4}\left(3 g^{2}+g^{\prime 2}\right) L_{b}\right.\right. \\
\left.\left.-3 y_{t}^{2} L_{f}\right)\right] \\
\left(\Sigma^{a} \Sigma^{a}\right)_{3 \mathrm{~d}}=\frac{\left(\Sigma^{a} \Sigma^{a}\right)_{4 \mathrm{~d}}}{T}\left[1-\frac{1}{(4 \pi)^{2}}\left(6 g^{2} L_{b}\right)\right]
\end{array}
$$

\section{Matching relations between superheavy and heavy scales}

Below, we list the matching relations between the superheavy and heavy scales. The formulas below are quoted only to the level of accuracy needed to consistently achieve matching relations at the light scale in the next subsection to $\mathcal{O}\left(g^{4}\right)$.

The Debye masses for the SU(2), U(1), and SU(3) gauge fields, respectively, are

$$
\begin{gathered}
m_{D}^{2}=g^{2} T^{2}\left(\frac{4+N_{d}+2 N_{t}}{6}+\frac{N_{f}}{3}\right) \\
m_{D}^{\prime 2}=g^{\prime 2} T^{2}\left(\frac{N_{d}}{6}+\frac{5 N_{f}}{9}\right) \\
m_{D}^{\prime \prime 2}=g_{s}^{2} T^{2}\left(1+\frac{N_{f}}{6}\right) .
\end{gathered}
$$

Matching relations for the $\mathrm{SU}(2)$ and $\mathrm{U}(1)$ gauge coupling constants are

$$
\begin{aligned}
g_{3}^{2}= & g^{2}(\Lambda) T\left[1+\frac{g^{2}}{(4 \pi)^{2}}\left(\frac{44-N_{d}-2 N_{t}}{6} L_{b}\right.\right. \\
& \left.\left.+\frac{2}{3}-\frac{4 N_{f}}{3} L_{f}\right)\right] \\
g_{3}^{\prime 2}= & g^{\prime 2}(\Lambda) T\left[1+\frac{g^{\prime 2}}{(4 \pi)^{2}}\left(-\frac{N_{d}}{6} L_{b}-\frac{20 N_{f}}{9} L_{f}\right)\right] .
\end{aligned}
$$

The couplings between temporal and fundamental/ adjoint scalar fields are

$$
\begin{gathered}
h_{3}=\frac{g^{2}(\Lambda) T}{4} \\
h_{3}^{\prime}=\frac{g^{\prime 2}(\Lambda) T}{4} \\
h_{3}^{\prime \prime}=\frac{g(\Lambda) g^{\prime}(\Lambda) T}{2} \\
\omega_{3}=-\frac{2 T}{16 \pi^{2}} g_{s}^{2} y_{t}^{2}, \\
\delta_{3}=\frac{1}{2} g^{2}(\Lambda) T \\
\delta_{3}^{\prime}=-\frac{1}{2} g^{2}(\Lambda) T .
\end{gathered}
$$

The matching relations for quartic couplings of the scalar potential are

$$
\begin{aligned}
\lambda_{3}= & T\left\{\lambda(\Lambda)+\frac{1}{(4 \pi)^{2}}\left[\frac{1}{8}\left(3 g^{4}+g^{4}+2 g^{2} g^{\prime 2}\right)\right.\right. \\
+ & 3 L_{f}\left(y_{t}^{4}-2 \lambda y_{t}^{2}\right)-L_{b}\left(\frac{3}{16}\left(3 g^{4}+g^{\prime 4}+2 g^{2} g^{\prime 2}\right)\right. \\
& \left.\left.\left.-\frac{3}{2}\left(3 g^{2}+g^{\prime 2}-8 \lambda\right) \lambda+\frac{3}{4} a_{2}^{2}\right)\right]\right\}, \\
a_{2,3}= & T\left\{a_{2}(\Lambda)+\frac{1}{(4 \pi)^{2}}\left[2 g^{4}-3 a_{2} y_{t}^{2} L_{f}\right.\right. \\
& \quad-L_{b}\left(2 a_{2}^{2}+5 a_{2} b_{4}+3 g^{4}+6 a_{2} \lambda\right. \\
& \left.\left.\left.\quad-\frac{3}{4} a_{2}\left(g^{\prime 2}+11 g^{2}\right)\right)\right]\right\} \\
b_{4,3}= & T\left\{b_{4}(\Lambda)+\frac{1}{(4 \pi)^{2}}\left[4 g^{4}-L_{b}\left(a_{2}^{2}+11 b_{4}^{2}\right.\right.\right. \\
& \left.\left.\left.-12 g^{2} b_{4}+6 g^{4}\right)\right]\right\} .
\end{aligned}
$$

The matching relations for the mass parameters of the scalar potential are

$$
\begin{aligned}
\mu_{3}^{2}= & \left(\mu_{3}^{2}\right)_{\mathrm{SM}}+\frac{T^{2}}{8} a_{2}(\Lambda)+\frac{1}{16 \pi^{2}}\left\{+\frac{3}{2} a_{2} \mu_{\Sigma}^{2} L_{b}+T^{2}\left(\frac{5}{24} g^{4}+\frac{1}{2} a_{2} g^{2}-\frac{3}{8} a_{2} y_{t}^{2} L_{f}+L_{b}\left(-\frac{7}{16} g^{4}-\frac{5}{8} a_{2}^{2}-\frac{5}{8} a_{2} b_{4}+\frac{33}{32} a_{2} g^{2}\right.\right.\right. \\
& \left.\left.\left.+\frac{3}{32} a_{2} g^{\prime 2}-\frac{3}{4} a_{2} \lambda\right)+\frac{1}{T^{2}}\left(c+\ln \left(\frac{3 T}{\Lambda_{3 d}}\right)\right)\left(-\frac{3}{2} a_{2,3}^{2}+6 a_{2,3} g_{3}^{2}-\frac{3}{4} g_{3}^{4}\right)\right)\right\}
\end{aligned}
$$

where (see Refs. [4,43]) 


$$
\begin{aligned}
\left(\mu_{3}^{2}\right)_{\mathrm{SM}}= & -\mu^{2}(\Lambda)+\frac{T^{2}}{16}\left(3 g^{2}(\Lambda)+g^{\prime 2}(\Lambda)+4 y_{t}^{2}(\Lambda)+8 \lambda(\Lambda)\right)+\frac{1}{16 \pi^{2}}\left\{-\mu^{2}\left(\left(\frac{3}{4}\left(3 g^{2}+g^{\prime 2}\right)-6 \lambda\right) L_{b}-3 y_{t}^{2} L_{f}\right)\right. \\
& +T^{2}\left[\frac{167}{96} g^{4}+\frac{1}{288} g^{\prime 4}-\frac{3}{16} g^{2} g^{\prime 2}+\frac{1}{4} \lambda\left(3 g^{2}+g^{\prime 2}\right)+L_{b}\left(\frac{17}{16} g^{4}-\frac{5}{48} g^{\prime 4}-\frac{3}{16} g^{2} g^{\prime 2}+\frac{3}{4} \lambda\left(3 g^{2}+g^{\prime 2}\right)-6 \lambda^{2}\right)\right. \\
& +\frac{1}{T^{2}}\left(c+\ln \left(\frac{3 T}{\Lambda_{3 d}}\right)\right)\left(\frac{39 g_{3}^{4}}{16}-\frac{5 g_{3}^{\prime 4}}{16}-\frac{9}{8} g_{3}^{2} g_{3}^{\prime 2}+12 g_{3}^{2} h_{3}-6 h_{3}^{2}-2 h_{3}^{\prime 2}-3 h_{3}^{\prime \prime 2}+3 \lambda_{3}\left(3 g_{3}^{2}+g_{3}^{\prime 2}\right)-12 \lambda_{3}^{2}\right) \\
& -y_{t}^{2}\left(\frac{3}{16} g^{2}+\frac{11}{48} g^{\prime 2}+2 g_{s}^{2}\right)+\left(\frac{1}{12} g^{4}+\frac{5}{108} g^{\prime 4}\right) N_{f}+L_{f}\left(y_{t}^{2}\left(\frac{9}{16} g^{2}+\frac{17}{48} g^{\prime 2}+2 g_{s}^{2}-3 \lambda\right)+\frac{3}{8} y_{t}^{4}\right. \\
& \left.\left.\left.-\left(\frac{1}{4} g^{4}+\frac{5}{36} g^{\prime 4}\right) N_{f}\right)+\ln (2)\left(y_{t}^{2}\left(-\frac{21}{8} g^{2}-\frac{47}{72} g^{2}+\frac{8}{3} g_{s}^{2}+9 \lambda\right)-\frac{3}{2} y_{t}^{4}+\left(\frac{3}{2} g^{4}+\frac{5}{6} g^{\prime 4}\right) N_{f}\right)\right]\right\},
\end{aligned}
$$

and

$$
\begin{aligned}
\mu_{\Sigma, 3}^{2}= & -\mu_{\Sigma}^{2}+T^{2}\left(\frac{1}{6} a_{2}(\Lambda)+\frac{5}{12} b_{4}(\Lambda)+\frac{1}{2} g^{2}(\Lambda)\right)+\frac{1}{16 \pi^{2}}\left\{-\left(6 g^{2}-5 b_{4}\right) \mu_{\Sigma}^{2} L_{b}+2 \mu^{2} a_{2} L_{b}+T^{2}\left[\left(\frac{71}{18}+\frac{2}{9} N_{f}\right) g^{4}\right.\right. \\
& +\frac{5}{3} b_{4} g^{2}+\frac{1}{4} a_{2} g^{2}+\frac{1}{12} a_{2} g^{\prime 2}+L_{b}\left(\frac{5}{12} g^{4}-\frac{3}{4} a_{2}^{2}-\frac{55}{12} b_{4}^{2}+\frac{11}{8} a_{2} g^{2}+\frac{1}{8} a_{2} g^{\prime 2}+5 b_{4} g^{2}-\frac{5}{6} a_{2} b_{4}-a_{2} \lambda\right) \\
& +\frac{1}{T^{2}}\left(c+\ln \left(\frac{3 T}{\Lambda_{3 d}}\right)\right)\left(-2 a_{2,3}^{2}-10 b_{4,3}^{2}+a_{2,3}\left(3 g_{3}^{2}+g_{3}^{\prime 2}\right)+20 b_{4,3} g_{3}^{2}-3 g_{3}^{4}+24 g_{3}^{2} \delta_{3}-24 \delta_{3}^{2}+8 g_{3}^{2} \delta_{3}^{\prime}-16 \delta_{3} \delta_{3}^{\prime}-16 \delta_{3}^{\prime 2}\right) \\
& \left.\left.-L_{f}\left(\frac{1}{2} a_{2} y_{t}^{2}+\frac{2}{3} g^{4} N_{f}\right)+\ln (2)\left(3 a_{2} y_{t}^{2}+4 g^{4} N_{f}\right)\right]\right\}
\end{aligned}
$$

\section{Matching relations between heavy and light scales}

Below, we list matching relations for 3D theory parameters at the light scale, in which the heavy time components of the gauge fields $B_{0}, W_{0}^{a}$, and $G_{0}^{A}$ are integrated out, assuming that both the Higgs doublet and triplet mass parameters are light.

The relations are all valid to $\mathcal{O}\left(g^{4}\right)$, provided we appropriately drop higher-order terms arising from coupling constant products and also from $\mu_{3}$ and $\mu_{\Sigma, 3}$ inside the logarithms. We confirm that to the order calculated these relations are explicitly independent of the gauge parameter $\xi$,

$$
\begin{aligned}
& \bar{g}_{3}^{2}=g_{3}^{2}\left(1-\frac{g_{3}^{2}}{24 \pi m_{D}}\right), \\
& \bar{g}_{3}^{\prime 2}=g_{3}^{\prime 2} \\
& \bar{\lambda}_{3}=\lambda_{3}-\frac{1}{8 \pi}\left(\frac{3 h_{3}^{2}}{m_{D}}+\frac{h_{3}^{\prime 2}}{m_{D}^{\prime}}+\frac{h_{3}^{\prime 2}}{m_{D}+m_{D}^{\prime}}\right), \\
& \bar{a}_{2,3}=a_{2,3}-\frac{h_{3}}{2 \pi m_{D}}\left(3 \delta_{3}+\delta_{3}^{\prime}\right) \\
& \bar{b}_{4,3}=b_{4,3}-\frac{1}{2 \pi m_{D}}\left(3 \delta_{3}^{2}+2 \delta_{3} \delta_{3}^{\prime}+\delta_{3}^{2}\right), \\
& \bar{\mu}_{3}^{2}=\mu_{3}^{2}-\frac{1}{4 \pi}\left(3 h_{3} m_{D}+h_{3}^{\prime} m_{D}^{\prime}+8 \omega_{3} m_{D}^{\prime \prime}\right)+\frac{1}{16 \pi^{2}}\left(3 g_{3}^{2} h_{3}-3 h_{3}^{2}-h_{3}^{\prime 2}-\frac{3}{2} h_{3}^{\prime \prime 2}+2 \mu_{3}\left(3 \frac{h_{3}^{2}}{m_{D}}+\frac{h_{3}^{\prime 2}}{m_{D}^{\prime}}\right)\right. \\
& +\left(-\frac{3}{4} g_{3}^{4}+12 g_{3}^{2} h_{3}\right) \ln \left(\frac{\Lambda_{3 d}}{2 m_{D}}\right)-6 h_{3}^{2} \ln \left(\frac{\Lambda_{3 d}}{2 m_{D}+\mu_{3}}\right)-2 h_{3}^{\prime 2} \ln \left(\frac{\Lambda_{3 d}}{2 m_{D}^{\prime}+\mu_{3}}\right) \\
& \left.-3 h_{3}^{\prime \prime 2} \ln \left(\frac{\Lambda_{3 d}}{m_{D}+m_{D}^{\prime}+\mu_{3}}\right)\right)+\frac{3}{16 \pi^{2}} h_{3}\left(3 \delta_{3}+\delta_{3}^{\prime}\right) \frac{\mu_{\Sigma, 3}}{m_{D}},
\end{aligned}
$$




$$
\begin{aligned}
\bar{\mu}_{\Sigma, 3}^{2}= & \mu_{\Sigma, 3}^{2}+\frac{m_{D}}{2 \pi}\left(3 \delta_{3}+\delta_{3}^{\prime}\right)+\frac{1}{16 \pi^{2}}\left(2 g_{3}^{2}\left(3 \delta_{3}+\delta_{3}^{\prime}\right)-12 \delta_{3}^{2}-12 \delta_{3} \delta_{3}^{\prime}-8 \delta_{3}^{\prime 2}+4 h_{3}\left(3 \delta_{3}+\delta_{3}^{\prime}\right) \frac{\mu_{3}}{m_{D}}\right. \\
& +\left(18 \delta_{3}^{2}+12 \delta_{3} \delta_{3}^{\prime}+2 \delta_{3}^{\prime 2}\right) \frac{\mu_{\Sigma, 3}}{m_{D}}+\left(-2 g_{3}^{4}+8 g_{3}^{2}\left(3 \delta_{3}+\delta_{3}^{\prime}\right)\right) \ln \left(\frac{\Lambda_{3 d}}{2 m_{D}}\right) \\
& \left.-\left(24 \delta_{3}^{2}+24 \delta_{3} \delta_{3}^{\prime}+16 \delta_{3}^{\prime 2}\right) \ln \left(\frac{\Lambda_{3 d}}{2 m_{D}+\mu_{\Sigma, 3}}\right)\right) .
\end{aligned}
$$

\section{Matching relations between heavy and light scales where the heavy triplet is integrated out}

Below, we list matching relations for the light scale DR3EFT parameters, in which the zero Matsubara mode of the real triplet $\Sigma$ is integrated out simultaneously with the time components of the gauge fields $B_{0}, W_{0}^{a}$, and $G_{0}^{A}$ :

$$
\begin{gathered}
\bar{g}_{3}^{2}=g_{3}^{2}\left(1-\frac{g_{3}^{2}}{24 \pi}\left(\frac{1}{\mu_{\Sigma, 3}}+\frac{1}{m_{D}}\right)\right), \\
\bar{g}_{3}^{\prime 2}=g_{3}^{\prime 2}, \\
\bar{\lambda}_{3}=\lambda_{3}-\frac{1}{8 \pi}\left(\frac{3 h_{3}^{2}}{m_{D}}+\frac{h_{3}^{\prime 2}}{m_{D}^{\prime}}+\frac{h_{3}^{\prime \prime 2}}{m_{D}+m_{D}^{\prime}}+\frac{3 a_{2,3}^{2}}{4 \mu_{\Sigma, 3}}\right), \\
\bar{\mu}_{3}^{2}=\mu_{3}^{2}-\frac{1}{4 \pi}\left(3 h_{3} m_{D}+h_{3}^{\prime} m_{D}^{\prime}+\frac{3 a_{2,3} \mu_{\Sigma, 3}}{2}\right) \\
+\frac{1}{16 \pi^{2}}\left\{\left(3 g_{3}^{2} h_{3}-3 h_{3}^{2}-h_{3}^{\prime 2}-\frac{3}{2} h_{3}^{\prime \prime 2}+2 \mu_{3}\left(3 \frac{h_{3}^{2}}{m_{D}}+\frac{h_{3}^{\prime 2}}{m_{D}^{\prime}}\right)\right.\right. \\
+\left(-\frac{3}{4} g_{3}^{4}+12 g_{3}^{2} h_{3}\right) \ln \left(\frac{\Lambda_{3 d}}{2 m_{D}}\right)-6 h_{3}^{2} \ln \left(\frac{\Lambda_{3 d}}{2 m_{D}+\mu_{3}}\right) \\
\left.+2 h_{3}^{\prime 2} \ln \left(\frac{\Lambda_{3 d}}{2 m_{D}^{\prime}+\mu_{3}}\right)-3 h_{3}^{\prime \prime 2} \ln \left(\frac{\Lambda_{3 d}}{m_{D}+m_{D}^{\prime}+\mu_{3}}\right)\right\}_{\mathrm{SM}} \\
+\frac{1}{16 \pi^{2}}\left\{\left(\frac{3 h_{3} \mu_{\Sigma, 3}^{2}}{m_{D}}+\frac{3 a_{2,3} m_{D}}{2 \mu_{\Sigma, 3}^{2}}\right)\left(3 \delta_{3}+\delta_{3}^{\prime}\right)-\frac{3}{4} a_{2,3}^{2}+\frac{15}{4} a_{2,3}^{2} b_{4,3}\right. \\
\left.+\frac{3}{2} a_{2,3} g_{3}^{2}+\frac{3}{2} a_{2,3}^{2} \frac{\mu_{3}}{\mu_{\Sigma, 3}}+\left(6 a_{2,3} g_{3}^{2}-\frac{3}{4} g_{3}^{4}\right) \ln \left(\frac{\Lambda_{3 d}}{2 \mu_{\Sigma, 3}}\right)-\frac{3}{2} a_{2,3}^{2} \ln \left(\frac{\Lambda_{3 d}}{2 \mu_{\Sigma, 3}+\mu_{3}}\right)\right\}_{\Sigma \mathrm{SM}}
\end{gathered} .
$$

\section{APPENDIX B: MATCHING RELATIONS IN THE CASE OF SUPERHEAVY TRIPLET}

In the case in which the mass parameter of the real triplet is large and negative, $\left|\mu_{\Sigma}^{2}\right| \gtrsim(\pi T)^{2}$, the real triplet d.o.f. are classified as superheavy, and all their Matsubara modes (including the zero mode) are integrated out to derive the heavyscale DR3EFT. Matching relations for parameters of the resulting 3D theory require the evaluation sum integrals involving the real triplet. Because the two-loop sum integrals are technically difficult, we have carried out the matching to only $\mathcal{O}\left(g^{2}\right)$ for the mass parameters $\mu_{3}^{2}$ and $\mu_{\Sigma, 3}^{2}$. The relations below are written in terms of derivatives of the bosonic thermal function

$$
J_{B}\left(z^{2}\right)=\int_{0}^{\infty} d x x^{2} \ln \left(1-e^{-\sqrt{x^{2}+z^{2}}}\right)
$$

evaluated at $z^{2}=\left|\mu_{\Sigma}^{2}\right| / T^{2}$.

The normalizations of the SU(2) gauge fields are

$W_{3 \mathrm{~d}, 0}^{2}=\frac{W_{4 \mathrm{~d}, 0}^{2}}{T}\left[1+\frac{g^{2}}{(4 \pi)^{2}}\left(\frac{N_{d}-26}{6} L_{b}+\frac{1}{3}\left(8+N_{d}\right)+\frac{4 N_{f}}{3}\left(L_{f}-1\right)+\frac{N_{t}}{3}\left(16 \pi^{2}\left(\frac{-1}{\pi^{2}} J_{B}^{\prime \prime}+\frac{4 \mu_{\Sigma}^{2}}{2 \pi^{2} T^{2}} J_{B}^{\prime \prime \prime}+\ln \left|\frac{\Lambda^{2}}{\mu_{\Sigma}^{2}}\right|\right)\right)\right]\right.$, 


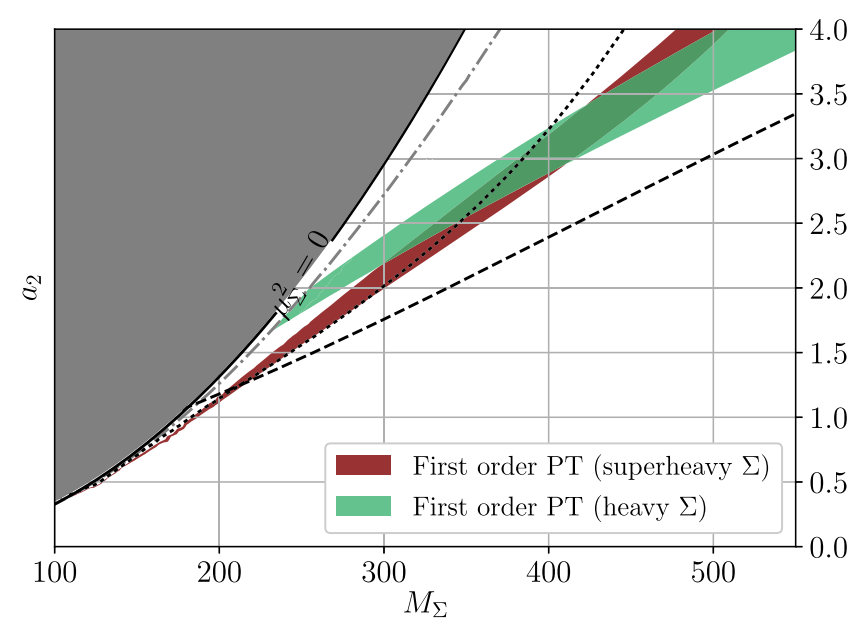

FIG. 5. Comparison of heavy and superheavy $\Sigma$ approximations. The gray dotted-dashed line shows the loop-corrected $\mu_{\Sigma}^{2}=0$ curve, and the black dashed and dotted curves show $T_{c}=140 \mathrm{GeV}$ for the heavy and superheavy cases, respectively.

$$
\vec{W}_{3 \mathrm{~d}}^{2}=\frac{\vec{W}_{4 \mathrm{~d}}^{2}}{T}\left[1+\frac{g^{2}}{(4 \pi)^{2}}\left(\frac{N_{d}-26}{6} L_{b}-\frac{2}{3}+\frac{4 N_{f}}{3} L_{f}+\frac{N_{t}}{3}\left(-16 J_{B}^{\prime \prime}+\ln \left|\frac{\Lambda^{2}}{\mu_{\Sigma}^{2}}\right|\right)\right)\right] .
$$

Normalizations of all other fields do not depend on the real triplet and are therefore identical to those listed in Eqs. (A6)-(A8).

The parameters of the heavy-scale DR3EFT that are modified by superheavy triplet are listed below. And other relations remain the same as in the earlier section:

$$
\begin{aligned}
& m_{D}^{2}=g^{2} T^{2}\left(\frac{4+N_{d}}{6}+\frac{N_{f}}{3}+\frac{4 N_{t}}{\pi^{2}}\left(J_{B}^{\prime}+\frac{\mu_{\Sigma}^{2}}{T^{2}} J_{B}^{\prime \prime}\right)\right) \\
& g_{3}^{2}=g^{2}(\Lambda) T\left[1+\frac{g^{2}}{(4 \pi)^{2}}\left(\frac{44-N_{d}}{6} L_{b}+\frac{2}{3}-\frac{4 N_{f}}{3} L_{f}-\frac{N_{t}}{3}\left(-16 J_{B}^{\prime \prime}+\ln \left|\frac{\Lambda^{2}}{\mu_{\Sigma}^{2}}\right|\right)\right)\right], \\
& \kappa_{3}=T g^{4}\left[\frac{1}{16 \pi^{2}}\left(\frac{16+N_{d}-4 N_{f}}{3}\right)+\frac{8\left(\mu_{\Sigma}^{2}\right)^{2}}{3 \pi^{2} T^{4}} J_{B}^{\prime \prime \prime \prime}\right], \\
& h_{3}=\frac{g^{2}(\Lambda) T}{4}\left(1+\frac{1}{(4 \pi)^{2}}\left\{\left[\frac{44-N_{d}}{6} L_{b}+\frac{53}{6}-\frac{N_{d}}{3}-\frac{4 N_{f}}{3}\left(L_{f}-1\right)\right.\right.\right. \\
& \left.\left.\left.-\frac{N_{t}}{3}\left(-16\left(J_{B}^{\prime \prime}+\frac{2 \mu_{\Sigma}^{2}}{\pi^{2} T^{2}} J_{B}^{\prime \prime \prime}\right)+\ln \left|\frac{\Lambda^{2}}{\mu_{\Sigma}^{2}}\right|\right)\right] g^{2}+\frac{g^{\prime 2}}{2}-6 y_{t}^{2}+12 \lambda+128 a_{2} \frac{\mu_{\Sigma}^{2}}{T^{2}} J_{B}^{\prime \prime \prime}\right\}\right), \\
& \mu_{3}^{2}=-\mu^{2}(\Lambda)+T^{2}\left(\frac{1}{16}\left(3 g^{2}(\Lambda)+g^{2}(\Lambda)\right)+\frac{1}{4} y_{t}^{2}(\Lambda)+\frac{1}{2} \lambda(\Lambda)\right)+\frac{3}{2} a_{2}(\Lambda)\left(\frac{T^{2}}{\pi^{2}} J_{B}^{\prime}+\frac{\mu_{\Sigma}^{2}}{16 \pi^{2}}\left(1+\ln \left|\frac{\Lambda^{2}}{\mu_{\Sigma}^{2}}\right|\right)\right), \\
& \lambda_{3}=T\left\{\lambda(\Lambda)+\frac{1}{(4 \pi)^{2}}\left[\frac{1}{8}\left(3 g^{4}+g^{4}+2 g^{2} g^{\prime 2}\right)+3 L_{f}\left(y_{t}^{4}-2 \lambda y_{t}^{2}\right)\right.\right. \\
& \left.\left.-L_{b}\left(\frac{3}{16}\left(3 g^{4}+g^{\prime 4}+2 g^{2} g^{\prime 2}\right)-\frac{3}{2}\left(3 g^{2}+g^{2}-8 \lambda\right) \lambda\right)-\frac{3}{4} a_{2}^{2}\left(-16 J_{B}^{\prime}+\ln \left|\frac{\Lambda^{2}}{\mu_{\Sigma}^{2}}\right|\right)\right]\right\} .
\end{aligned}
$$

Matching relations for the parameters of the light-scale DR3EFT for the superheavy triplet remain the same as in Appendix A 4 above.

In Fig. 5, we show a comparison between heavy and superheavy approximations. The first-order transition region is again given by 3D parameter $0<x<0.11$. Black dashed and dotted curves show $T_{c}=140 \mathrm{GeV}$ for heavy and superheavy 
cases, respectively. We observe that locations of first-order regions agree qualitatively, while $T_{c}$ curves show a larger discrepancy. We assume that this difference in critical temperatures is related to our approximation in the superheavy case, in which we only used one-loop level determination for the mass parameter that gives $y$, from which $T_{c}$ is solved.

\section{APPENDIX C: COUNTERTERMS OF THE 3D EFFECTIVE THEORIES}

In this section, we collect the counterterms associated with the logarithmic UV divergences of the 3D effective theory. The UV-divergent parts can be extracted by a direct diagrammatic calculation of the scalar self-energies at zero external momentum at two loops. At the DR3EFT at the heavy scale, the mass parameter counterterm for the doublet is

$$
\delta \mu_{3}^{2}=\left(\delta \mu_{3}^{2}\right)_{\mathrm{SM}}-\frac{1}{16 \pi^{2}} \frac{1}{4 \epsilon}\left(-\frac{3}{4} g_{3}^{4}+6 a_{2,3} g_{3}^{2}-\frac{3}{2} a_{2,3}^{2}\right),
$$

where the pure Standard Model contribution is

$$
\left(\delta \mu_{3}^{2}\right)_{\mathrm{SM}}=-\frac{1}{16 \pi^{2}} \frac{1}{4 \epsilon}\left(\frac{39}{16} g_{3}^{4}+12 g_{3}^{2} h_{3}-6 h_{3}^{2}+9 g_{3}^{2} \lambda_{3}-12 \lambda_{3}^{2}-\frac{5}{16} g_{3}^{\prime 4}-\frac{9}{8} g_{3}^{2} g_{3}^{\prime 2}-2 h_{3}^{\prime 2}-3 h_{3}^{\prime \prime 2}+3 g_{3}^{\prime 2} \lambda_{3}\right)
$$

and the mass parameter counterterm for the real triplet is

$\delta \mu_{\Sigma, 3}^{2}=-\frac{1}{16 \pi^{2}} \frac{1}{4 \epsilon}\left(-3 g_{3}^{4}+8 g_{3}^{2}\left(3 \delta_{3}+\delta_{3}^{\prime}\right)-8\left(3 \delta_{3}^{2}+2 \delta_{3} \delta_{3}^{\prime}+2 \delta_{3}^{\prime 2}\right)+a_{2,3}\left(3 g_{3}^{2}+g_{3}^{\prime 2}\right)+20 b_{4,3} g_{3}^{2}-2 a_{2,3}^{2}-10 b_{4,3}^{2}\right)$.

In the DR3EFT at the light scale, the mass parameter counterterm for the doublet is

$$
\delta \bar{\mu}_{3}^{2}=\delta \bar{\mu}_{3}^{2, \mathrm{SM}}-\frac{1}{16 \pi^{2}} \frac{1}{4 \epsilon}\left(-\frac{3}{4} \bar{g}_{3}^{4}+6 \bar{a}_{2,3} \bar{g}_{3}^{2}-\frac{3}{2} \bar{a}_{2,3}^{2}\right),
$$

where the Standard Model contribution is

$$
\delta \bar{\mu}_{3}^{2, \mathrm{SM}}=-\frac{1}{16 \pi^{2}} \frac{1}{4 \epsilon}\left(\frac{51}{16} \bar{g}_{3}^{4}+9 \bar{g}_{3}^{2} \bar{\lambda}_{3}-12 \bar{\lambda}_{3}^{2}-\frac{5}{16} \bar{g}_{3}^{\prime 4}-\frac{9}{8} \bar{g}_{3}^{2}{\overline{g_{3}^{\prime}}}^{2}+3 \bar{g}_{3}^{\prime 2} \bar{\lambda}_{3}\right),
$$

and the mass parameter counterterm for the real triplet is

$$
\delta \bar{\mu}_{\Sigma, 3}^{2}=-\frac{1}{16 \pi^{2}} \frac{1}{4 \epsilon}\left(-\bar{g}_{3}^{4}+\bar{a}_{2,3}\left(3 \bar{g}_{3}^{2}+\bar{g}_{3}^{\prime 2}\right)+20 \bar{b}_{4,3} \bar{g}_{3}^{2}-2 \bar{a}_{2,3}^{2}-10 \bar{b}_{4,3}^{2}\right) .
$$

[1] P. A. R. Ade et al. (Planck Collaboration), Astron. Astrophys. 594, A13 (2016).

[2] A. D. Sakharov, Pis'ma Zh. Eksp. Teor. Fiz. 5, 32 (1967); Usp. Fiz. Nauk 161, 61 (1991).

[3] D. E. Morrissey and M. J. Ramsey-Musolf, New J. Phys. 14, 125003 (2012).

[4] K. Kajantie, M. Laine, K. Rummukainen, and M. E. Shaposhnikov, Nucl. Phys. B458, 90 (1996).

[5] M. Gurtler, E.-M. Ilgenfritz, and A. Schiller, Phys. Rev. D 56, 3888 (1997).

[6] M. Laine and K. Rummukainen, Nucl. Phys. B, Proc. Suppl. 73, 180 (1999).
[7] F. Csikor, Z. Fodor, and J. Heitger, Phys. Rev. Lett. 82, 21 (1999).

[8] K. Rummukainen, M. Tsypin, K. Kajantie, M. Laine, and M. E. Shaposhnikov, Nucl. Phys. B532, 283 (1998).

[9] Y. Aoki, F. Csikor, Z. Fodor, and A. Ukawa, Phys. Rev. D 60, 013001 (1999).

[10] S. Chatrchyan et al. (CMS Collaboration), Phys. Lett. B 716, 30 (2012).

[11] G. Aad et al. (ATLAS Collaboration), Phys. Lett. B 716, 1 (2012).

[12] J. R. Espinosa and M. Quiros, Phys. Lett. B 305, 98 (1993).

[13] J. Choi and R. R. Volkas, Phys. Lett. B 317, 385 (1993). 
[14] S. W. Ham, Y. S. Jeong, and S. K. Oh, J. Phys. G 31, 857 (2005).

[15] S. Profumo, M. J. Ramsey-Musolf, and G. Shaughnessy, J. High Energy Phys. 08 (2007) 010.

[16] J. R. Espinosa, T. Konstandin, and F. Riva, Nucl. Phys. B854, 592 (2012).

[17] J. M. Cline and K. Kainulainen, J. Cosmol. Astropart. Phys. 01 (2013) 012.

[18] A. V. Kotwal, M. J. Ramsey-Musolf, J. M. No, and P. Winslow, Phys. Rev. D 94, 035022 (2016).

[19] C.-Y. Chen, J. Kozaczuk, and I. M. Lewis, J. High Energy Phys. 08 (2017) 096.

[20] M. Jiang, L. Bian, W. Huang, and J. Shu, Phys. Rev. D 93, 065032 (2016).

[21] C.-W. Chiang, M. J. Ramsey-Musolf, and E. Senaha, Phys. Rev. D 97, 015005 (2018).

[22] A. Katz and M. Perelstein, J. High Energy Phys. 07 (2014) 108.

[23] A. Katz, M. Perelstein, M. J. Ramsey-Musolf, and P. Winslow, Phys. Rev. D 92, 095019 (2015).

[24] P. Fileviez Perez, H. H. Patel, M. Ramsey-Musolf, and K. Wang, Phys. Rev. D 79, 055024 (2009).

[25] H. H. Patel and M. J. Ramsey-Musolf, Phys. Rev. D 88, 035013 (2013).

[26] S. Inoue, G. Ovanesyan, and M. J. Ramsey-Musolf, Phys. Rev. D 93, 015013 (2016).

[27] N. Blinov, J. Kozaczuk, D. E. Morrissey, and C. Tamarit, Phys. Rev. D 92, 035012 (2015).

[28] J. O. Andersen, T. Gorda, A. Helset, L. Niemi, T. V. I. Tenkanen, A. Tranberg, A. Vuorinen, and D. J. Weir, Phys. Rev. Lett. 121, 191802 (2018).
[29] G. C. Dorsch, S. J. Huber, and J. M. No, J. High Energy Phys. 10 (2013) 029.

[30] P. Basler, M. Krause, M. Muehlleitner, J. Wittbrodt, and A. Wlotzka, J. High Energy Phys. 02 (2017) 121.

[31] G. C. Dorsch, S. J. Huber, K. Mimasu, and J. M. No, J. High Energy Phys. 12 (2017) 086.

[32] P. Basler, M. Mhlleitner, and J. Wittbrodt, J. High Energy Phys. 03 (2018) 061.

[33] D. J. Gross, R. D. Pisarski, and L. G. Yaffe, Rev. Mod. Phys. 53, 43 (1981).

[34] A. D. Linde, Phys. Lett. 96B, 289 (1980).

[35] M. Cirelli, N. Fornengo, and A. Strumia, Nucl. Phys. B753, 178 (2006).

[36] W. Chao, G.-J. Ding, X.-G. He, and M. J. Ramsey-Musolf (to be published).

[37] G. Aad et al. (ATLAS Collaboration), Phys. Rev. D 88, 112006 (2013).

[38] V. Khachatryan et al. (CMS Collaboration), J. High Energy Phys. 01 (2015) 096.

[39] T. Brauner, T. V. I. Tenkanen, A. Tranberg, A. Vuorinen, and D. J. Weir, J. High Energy Phys. 03 (2017) 007.

[40] J. Brandstetter (ATLAS and CMS Collaborations), in International Workshop on Future Linear Collider (LCWS2017) Strasbourg, France, 2017 (2018), http:// inspirehep.net/record/1650149/files/arXiv:1801.07926.pdf.

[41] C. Caprini et al., J. Cosmol. Astropart. Phys. 04 (2016) 001.

[42] H. H. Patel and M. J. Ramsey-Musolf, J. High Energy Phys. 07 (2011) 029.

[43] T. Gorda, A. Helset, L. Niemi, T. V. I. Tenkanen, and D. J. Weir, J. High Energy Phys. 02 (2019) 081. 